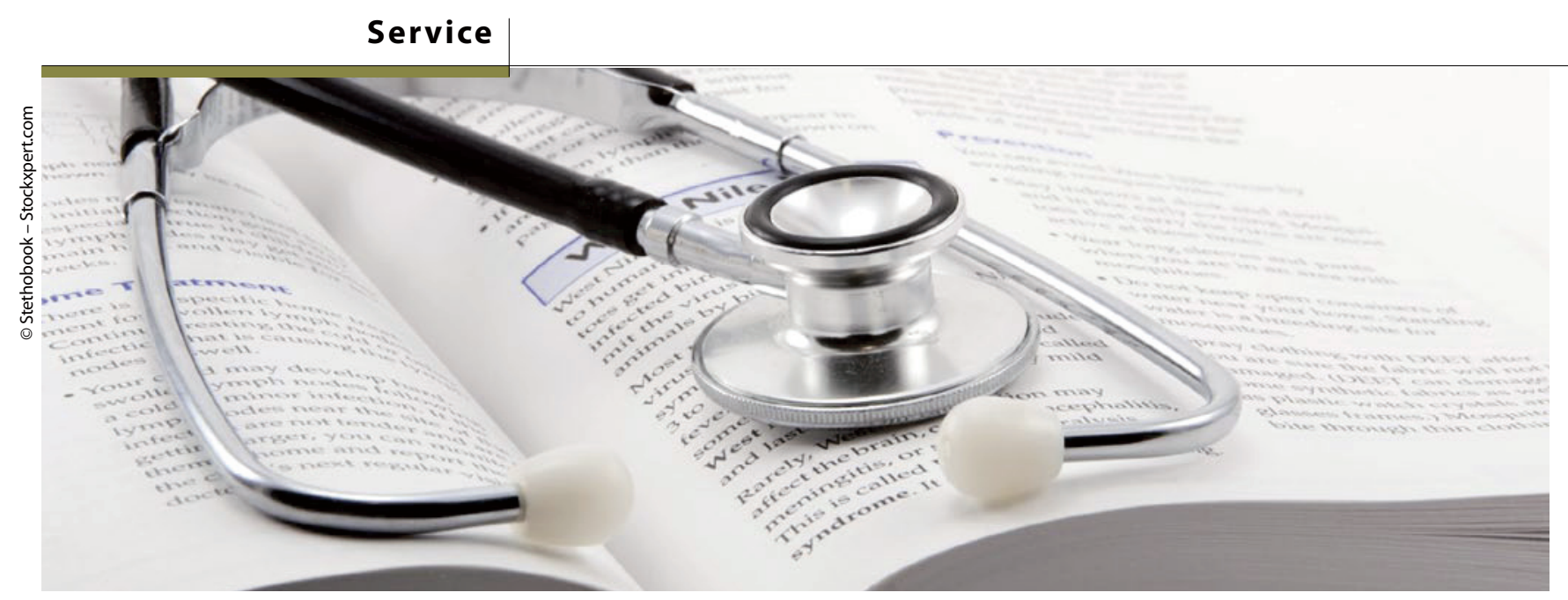

\title{
Medizinische Fortbildung
}

\section{Bücher im Fokus}

\section{Erfolg im Beruf, das bedeutet in der Arztpraxis zweierlei: fachlich auf dem aktuellen Stand zu sein und betriebswirtschaftlich ein gesundes Unternehmen zu führen. Wesentlich ist daher die stetige Fortbildung in der einen wie in der anderen Hinsicht, durch Kongresse, Workshops und nach wie vor durch Zeitschriften und Bücher.}

\section{Schmerztherapie - effektiv und patientenorientiert}

Die Autoren schildern den Weg vom Symptom zur Diagnose und zu einer effektiven und patientenorientierten Schmerztherapie. Ihr Buch enthält aktuelle Leitlinien, evidenzbasierte Therapieempfehlungen und zahlreiche Abbildungen. Alle Ärzte, die Patienten mit chronischen Schmerzen be-

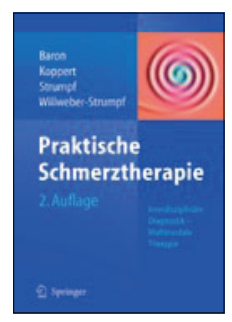

R. Baron, W. Koppert M. Strumpf, A.Willweber (Hrsg.)

Praktische Schmerztherapie Interdisziplinäre Diagnostik - multimodale Therapie Springer Verlag 2011 2. aktualisierte, erw. Aufl. ISBN: 978-3-642-12818-9 79,95 Euro

handeln erhalten klare Angaben zu spezifisch schmerztherapeutischen Vorgehensweisen und Verfahren sowie zur spezifischen Behandlung der verschiedenen chronischen Schmerzsyndrome.

Besonderes Augenmerk gilt der Darstellung der aktuellen Evidenz für den Einsatz der verschiedenen Verfahren als auch der evidenzbasierten Vorgehensweise bei der Behandlung einzelner Krankheitsbilder.

\section{Unternehmen Arztpraxis}

Wenn das "Unternehmen Arztpraxis" florieren soll, bilden die Aspekte Betriebswirtschaft, Kooperationsformen, Steuer-/Vertragsarzt-/ Arbeits-/Haftungsrecht, Marketing, Mitarbeiterführung, Praxisorganisation und auch die Altersvorsorge die Basis. Aber erst durch das sinnvolle Zusammenspiel und die Wechselwirkungen aller dieser Aspekte entsteht ein langfristiges Gesamtkonzept. Auch die enge Verbindung von Praxis- und Privatbereich ist von existenzieller Bedeutung. Dies führen die

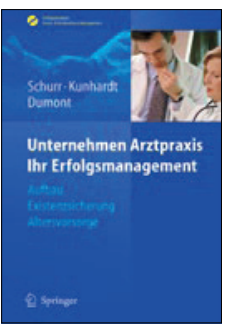

M. Schurr, H. Kunhardt M. Dumont Unternehmen Arztpraxis - Ihr Erfolgsmanagement Aufbau - Existenzsicherung - Altersvorsorge Springer Verlag 2009 ISBN: 978-3-540-48559-9 39,95 Euro

Autoren - alle drei erfahrene Experten auf ihrem Gebiet - klar verständlich vor Augen: nicht nur für Einsteiger, die eine Praxis gründen oder übernehmen wollen, sondern auch für Niedergelassene, die ihr Praxiskonzept optimieren und ihr "Management-Wissen" auffrischen und erweitern wollen.

\section{Palliative Care}

Das Kursbuch Palliative Care bietet praxisnah und zugleich theoretisch fundiert eine Einführung und vertieftes Wissen in

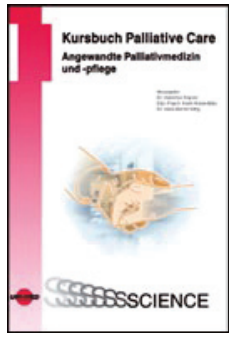

H. Kayser, K. Kieseritzky, H.-B. Sittig (Hrsg.) Kursbuch Palliative Care. Angewandte Palliativmedizin und -pflege UNI-MED Science 2009 ISBN 978-3-8374-1080-8 44,80 Euro

alle Aspekte von Palliative Care. Der Leser erhält einen Einblick in medizinische, pflegerische, psychische, soziale, ethische, rechtliche und spirituelle Aspekte der Versorgung schwerstkranker und sterbender Menschen. Umfassend und praxisnah wird die Symptomkontrolle dargestellt, ebenso die Behandlung durch das interdisziplinäre Team, Aspekte der Kommunikation und des eigenen Umgangs mit Tod und Sterben sowie neue Versorgungsstrukturen und Qualitätssicherung. Das Nachschlagewerk richtet sich an alle in diesem Bereich tätigen und interessierten Berufsgruppen und an Studenten. Es regt zudem zur vertieften Beschäftigung mit Fragen rund um Palliative Care an. 